\title{
THE VARIABLE STAR POPULATION OF NGC 1866
}

\author{
DOUGLAS L. WELCH, PATRICK CÔTÉ \\ Dept. of Physics, McMaster University \\ Hamilton, Ontario L8S $4 M 1$ \\ Canada \\ MARIO MATEO \\ Observatories of the Carnegie Institution of Washington \\ 813 Santa Barbara St, Pasadena, CA 91101 \\ USA \\ BARRY F. MADORE \\ Infrared Processing \& Analysis Center, Jet Propulsion Laboratory \\ M/S 100-22, California Institute of Technology \\ Pasadena, CA 91125 \\ USA
}

ABSTRACT. We report the results of an extensive photometric and radial velocity study of the variable stars in the Large Magellanic Cloud (LMC) cluster NGC 1866. We find:
1) twenty probable member Cepheid variables,
2) V lightcurve amplitude decreasing toward the red edge,
3) lightcurves, periods, and memberships for Storm-type variables,
4) one apparently stable star in the instability strip,
5) an overtone pulsator in the fundamental region of the strip,
6) a single-lined spectroscopic binary member Cepheid,
7) an essentially dispersionless P-L-C with $\beta=2.0 \pm 0.4$,
8) at least one red supergiant variable of long period, and
9) a true distance modulus of $18.57 \pm 0.01 \mathrm{mag}$ for the cluster.

\section{Introduction}

NGC 1866 is a well-known, young, Cepheid-rich cluster in the LMC. The importance of Cepheid variables as distance indicators and the promise of improved observational material and additional constraints on Cepheid structure and the instability strip led us to undertake a new CCD survey of this cluster. Our specific goals for this undertaking were: to discover variables in the cluster core, to discover low-amplitude variables if they existed, to confirm the variable identifications of Storm et al. (1988) and to establish periods and lightcurve parameters where possible, to investigate the amplitude-colour correlation, to determine the red edge of the instability strip by observation, to establish membership from radial velocities, and to provide data for a Baade-Wesselink analysis.

The existence of large numbers of Cepheid variables in a cluster with known distance allows a unique comparison of evolutionary models and theory for intermediate-mass stars. The Cepheids in NGC 1866 share, to good approximation, the same distance, reddening, metallicity, age and mass. 


\section{Observations}

\subsection{PHOTOMETRY}

Over 90 epochs of BV CCD photometry were obtained on the Las Campanas $1.0 \mathrm{~m}$, CTIO $4.0 \mathrm{~m}$ and $0.9 \mathrm{~m}$ telescopes over three years. Magnitudes were determined using ALLSTAR (Stetson 1987) and DoPHOT (Mateo \& Schechter 1989). While these routines are very good at estimating magnitudes in crowded fields, the centre 30 arcsec are still unusable due to seeing-dependent star lists and sky-annulus variations.

\subsection{RADIAL VELOCITIES}

Spectra of NGC 1866 stars were obtained during two runs (1988 December 16-24 and 1989 November 30-December 17) using the photon-counting échelle spectrograph on the $2.5 \mathrm{~m}$ Dupont reflector at Las Campanas, Chile. Each 3.1 Mbyte spectrum was reduced to a radial velocity using tasks in the échelle and $r v$ packages of IRAF. Velocities were determined relative to a local standard and IAU standards. Typically, the radial velocities have uncertainties of $\pm 1-2 \mathrm{~km} \mathrm{~s}^{-1}$. More than 100 velocities of NGC 1866 variables were obtained.

A velocity close to $300 \mathrm{~km} \mathrm{~s}^{-1}$ is found for cluster stars, the final value depending on details of reduction which will be discussed elsewhere. This is in contrast to the cluster velocity of $281 \pm 6$ $\mathrm{km} \mathrm{s}^{-1}$ adopted by Freeman et al. (1983).

\section{Conclusions}

The full details of observations, reductions and a more complete discussion will be submitted for publication elsewhere. The principal results are listed in the abstract and are not repeated here. The distance modulus was determined from the BV P-L-C relation reported by Walker (1987) in equation 3 of that paper. The extreme values of the true distance modulus, derived from nine uncrowded Cepheids with full phase coverage, are 18.52 and $18.65 \mathrm{mag}$, assuming $\mathrm{E}_{B-V}=0.06$ mag.

\section{References}

Freeman, K.C., Illingworth, G., Oemler, A. (1983), Astrophys. J. 272, 488.

Mateo, M., Schechter, P. (1989), Proceedings of the First ESO Data Analysis Workshop, in press.

Stetson, P.B. (1987), Publ. Astron. soc. Pac. 99, 191.

Storm, J., Andersen, J., Blecha, A., Walker, M.F. (1988), Astron. Astrophys. 190, L18.

Walker, A.R. (1987), M.N.R.A.S. 225, 627. 